\title{
Underwater Target Recognition Based on Line Spectrum and Support Vector Machine
}

\author{
Liu Jian \\ Electrical Engineering College \\ Naval University of Engineering, NUE \\ Wuhan, China \\ liujian1974@hotmail.com \\ He Yang \\ Electrical Engineering College \\ Naval University of Engineering, NUE \\ Wuhan, China \\ 15527674280@163.com
}

\author{
Liu Zhong \\ Electrical Engineering College \\ Naval University of Engineering, NUE \\ Wuhan, China \\ 1062269312@qq.com \\ Xiong Ying \\ Naval Architecture \& Power Engineering College \\ Naval University of Engineering, NUE \\ Wuhan, China \\ 1501369441@qq.com
}

\begin{abstract}
To increase the classification of underwater target recognition by ship radiated noise signals, the feature extraction method of line spectrum and classification algorithm of support vector machine (SVM) are adopted in the paper. The basic principles of power spectrum and SVM are briefly introduced at first. Then the signals of ship radiated are processed by the power spectrum estimation and the nonlinear least-square polynomial to fitting the continuous spectrum. The ultimate line spectrum is obtained through selecting peak and removes side lobe, and then extracts the feature of number and intensity of line spectrum from it. At last, the characteristic vector is established with normalization process and the signals are classified by SVM with parameter optimization. The simulation experiment results demonstrate that the ship radiated signals can be classified or recognized availably based on line spectrum feature and SVM. This paper presents a method of underwater target recognition and provides guidance for the research on multi-classes recognition algorithms.
\end{abstract}

Keywords-line spectrum; feature extraction; support vector machine ; target recognition; algorithms

\section{INTRODUCTION}

In the phase of underwater target classification recognition, feature extraction from ship radiated noise signals is the key step. Because of complexity of ocean environment and particularity of acoustic communication channel, to find a good extraction method which both can be represented the target's essence and satisfied the demand of underwater detection, is the hotspot and topic in the domain ${ }^{[1,2]}$. Therefore researchers analyse and study these original signals of ship radiated noise from different angles, and several common feature extraction methods are obtained, mainly including time-domain waveform structure feature extraction, signal spectrum estimation feature extraction and time-frequency analysis feature extraction etc. The feature extraction method of signal spectrum attracts much attention including feature method of line spectrum, feature method of envelope spectrum and feature method of spectrum graph etc.

Ship radiated noises are generally made up of hydrodynamic noise, mechanical noise and propeller cavitation noise. They are represented by the superposition of broadband noise of continuous spectrum and single-frequency noise of discontinuous spectrum. Among them, the mechanical noise and propeller cavitation noise are major ship radiated noises, and there are three periodic vibration sources to generate line spectrum in mechanical noise. The detection, tracking and recognition of line spectrum is of great importance in the use of sonar, and a lot of researches have been conduct, including the standard, the evaluation criteria and the generation mechanism of line spectrum etc. ${ }^{[3]}$ As the line spectrum has the following two features: First it contains concentrated and stable energy; Second line spectrum itself carries frequency information. So the feature of line spectrum is often regarded as the important basis of target parameter estimation and recognition.

Statistical Learning Theory $(\mathrm{SLT})^{[4]}$ is a specialized small-sample statistical theory, which builds a better theoretical framework for statistic pattern recognition when studying finite samples and the broader issue of Machine Learning (ML). and SLT also develops a pattern recognition method, namely Support Vector Machine (SVM). Due to its good generalization ability , SVM ${ }^{[5]}$, a new machine learning method proposed by Vapnik in 1995, shows many unique advantages in solving the problem of small-sample, nonlinear and high-dimensional pattern recognition, and can be promoted to other ML questions, like function fitting and so on. Currently, SLT and SVM have already become the new hotspot of ML in the world and have been widely applied to many fields ${ }^{[6]}$.

In the paper, the ship radiated noise is processed by the power spectrum analysis at first, using the nonlinear least-square polynomial to fitting and approximate the continuous spectrum, and gradually separated spectral components. Then correct waveform and extract the 
feature of number and intensity of line spectrum. The characteristic vector is established with normalization process. The original signals of measurement noise are divided into training part and testing part, and the task of signal classification and recognition can be completed by applying classification algorithm of SVM to classify the ship radiated noise signals and optimize parameter.

\section{ALGORITHM PRINCIPLE}

\section{A. Theoretical model of the power spectrum}

Signal power spectrum reflects many important features of the signal, it is an important part of signal process in areas such as sonar, radar and noise analysis that using the features of continuous spectrum and line spectrum of the power spectrum to process target automatic identification and classification ${ }^{[7,8]}$.

Ship radiated noise is represented by the superposition of line spectrum and continuous spectrum, periodic signal can be used as the model of line spectrum, and broadband stationary random process is used to fitting the model of continuous spectrum. If the amplitude feature is considered, local stationary process can also be used, whose result is basically the same. According to this model, the stochastic process $\{S(t)\}$ of ship radiation noise can be expressed as:

$$
\{S(t)\}=\left\{x(t)+\sum_{i=1}^{n} 1_{i}(t)\right\}
$$

Where $\left\{x_{X}(t)\right\}$ is broadband stationary random process and $l_{i}(t)$ is periodic signal whose initial phase is random, $i=1,2, \cdots, n$.The power spectrum of ship radiation noise is calculated by the equation as follow:

$$
\{S(f)\}=\lim _{T \rightarrow \infty} \frac{1}{T} E\left[\left|S_{K, T}(f)\right|^{2}\right]
$$

Where $T$ is the signal length of each section which is taken from the transformation of FFT, $E$ is the average of set, $K$ is the number of signal segment. Equation (2) is the strict definition of ship radiated noise spectrum in mathematical model, that is, to obtain an infinite number of signal segments and the time length of each signal segment tends to infinity. In practical application, only the average of set with a finite length and number can be obtained. Then it's unilateral power spectrum estimation is:

$$
G(f)=\frac{2}{n_{d} T} \sum_{K=1}^{n_{d}}\left|S_{K, T}(f)\right|^{2}
$$

Where $n_{d}$ is the average number.

\section{B. Power spectrum estimation method}

In this paper, the estimation method of classical cycle map is adopt, whose biggest advantage is FFT-based and fast computing speed. It regards the $\mathrm{N}$ observations of random sequence $x(n)$ as a sequence of finite energy and calculates the discrete FFT transform directly to obtain $X(k)$, then to squared its amplitude and divide by $\mathrm{N}$ as the true power spectrum estimation of sequence $x(n){ }^{[9]}$.

\section{Continuous Spectrum Feature fitting}

Through the study of structure of the power spectrum, it can be found that the structure of noise spectrum of similar signals especially emitted by the same target has a continuous spectrum with similar trend, the following analysis of this trend (i.e. outline of continuous spectrum).

Suppose we obtain the $\mathrm{X}, \mathrm{Y}$ from the signals of time-domain samples by the FFT transform, where $X=\left\{X_{i}\right\}(i=1,2 \cdots, N)$ is the N-point discrete frequency and $Y=\left\{y_{i}\right\}(i=1,2 \cdots, N)$ means the corresponding digital power spectrum. The relationship between $\mathrm{Y}$ and $\mathrm{X}$ is given by the ideal curve function $Y=f(X, C)$. Where $f()$ is ideal curve function and $C=\left(c_{1}, c_{2}, \cdots c_{m}\right)^{\mathrm{T}}$ is undetermined coefficient vector. Corresponding to different power spectra, if $f()$ is a given function, $C$ will have different values, which reflects the contour features of the signal continuous spectrum. The following non-linear polynomial function is used to fitting power spectrum of this article:

$$
y=f(x, C)=c_{1} x^{n}+c_{2} x^{n-1}+\cdots c_{n} x+c_{n+1}
$$

Specific fitting rank is determined according to the actual situation, the general option is 3-5 order or sub-fitting. After obtaining the fitting continuous spectrum feature curve, the power spectrum is subtracted continuous spectrum, and line spectrum components can be extracted in the remaining spectrum.

\section{Line Spectrum Feature Extraction}

Line spectrum is a higher amplitude threshold in narrower width thresholds. Although periodogram power spectrum estimation based on FFT has a fast computing speed, because the length of spectrum is longer, the dimension is too large and the class attribute is not obvious, it is not easy to extract the line spectrum feature directly and often requires multiple transformations to enhance the useful information.

The flattened spectrum is obtained after the above treatment. Since the peak can't be at the intermediate transition point, first we can remove intermediate points which are consecutive rising or falling and only keep inflection points to simplify waveform, i.e. to find the maxima. Specific methods are as follows: assume the spectral values of three consecutive points $\mathrm{k}-1, \mathrm{k}$ and $\mathrm{k}+1$ are respectively $y_{k-1}, y_{k}$ and $y_{k+1}$, whose first order differential are $\Delta y_{k}=y_{k+1}-y_{k}$ and $\Delta y_{k-1}=y_{k}-y_{k-1}$. If $\Delta y_{k} \cdot \Delta y_{k-1}>0, \quad y_{k}$ is the intermediate point, which can be removed; if $\Delta y_{k} \cdot \Delta y_{k-1}<0, y_{k}$ is the inflection point, and we should retain it and mark its frequency.

After getting the spectrum which is selected by the peaks, we scan the spectrum by line spectrum and process the correction of waveform to extract the spectral distribution, the specific determination method is: For any point $y_{k}$, choose a threshold value $\delta_{\text {gatel }}$, if $y_{k}-y_{k-1}>\delta_{\text {gate }}$ or $y_{k}-y_{k+1}>\delta_{\text {gate }}, y_{k}$ is the line spectrum, otherwise not.

In addition, because line spectrum may have a higher side lobe and if two line spectral are closely spaced, there may be superposition of side lobes to produce a higher 
point, both of which may be mistaken for line spectrum ${ }^{[10]}$, the specific situation shown in Fig. 1. Removing method is: For any point $y_{k}$, choose a threshold value $\delta_{\text {gate } 2}$, if $y_{k-2}-y_{k}>\delta_{\text {gate } 2}$ or $y_{k+2}-y_{k}>\delta_{\text {gate } 2}$ and its frequency interval $\Delta f$ is within a certain range, $y_{k}$ is not the line spectrum, which should be removed. The choices of $\delta_{\text {gate }}$ and $\delta_{\text {gate } 2}$ should be based on the specific situation. Thus the line spectrum distribution of noise can be obtained from the original signal of ship radiation noise.
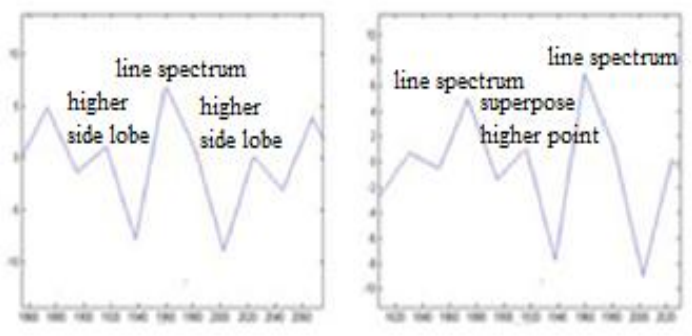

Figure 1. Sketch map of remove side lobe

After determining the components of line spectrum, it generate the desired original characteristic vector of line spectrum according to these line spectra. In this paper, we adopt the average segmentation method, first the frequency axis is evenly divided into $\mathrm{K}$ equal parts, which is divided into $\mathrm{K}$ frequency intervals, if the frequency of line spectrum falls in a frequency interval, the number and amplitude of line spectrum are cumulative until all line spectrum searches are completed. Then the number and average intensity of line spectrum are counted in each frequency interval to constitute the $2 \mathrm{~K}$-dimensional original characteristic vector.

\section{E. Support vector machine}

SVM is a classification method established on the SLT. It is mainly based on the following three considerations: (1) based on structural risk minimization, it has a strong ability to promote by minimizing the $\mathrm{VC}$ dimension of the functions set to control the structure risk of learning machine. (2) It is guaranteed by SLT related theorem to achieve the control of $\mathrm{VC}$ dimension by maximizing the classification interval (finding the optimal separating hyperplane). (3) The SVM is technically using nuclear technology, according to the functional Mercer theorem, it finds a function (called kernel function) to make the inner product in the sample space correspond to it in transform space, that is to avoid seeking the nonlinear mapping for the inner product ${ }^{[6]}$. SVM makes the input vector $\phi$ mapped to a high dimensional characteristic space by the pre-selected non-linear mapping $\phi$, where it constructs the optimal separating hyperplane, i.e. there is a hyperplane satisfying the conditions for the sample correct classification. Moreover, the distance of the nearest two points between the two sides of the hyperplane and the hyperplane is the maximum, so the nonlinear separable problem in the original space can be transformed into the linear separable problem in high-dimensional space.

Suppose the linear separable sample set is $\left(x_{i}, y_{i}\right), i=1, \cdots, l ; x_{i} \in R^{d}, y \in\{-1,+1\}$ is the class mark and $d$ is the space dimension. The optimal classification surface problem can be expressed as the following optimization problem, namely under the condition of constraint:

$$
y_{i}\left[\left(\omega \cdot x_{i}\right)-b\right]-1 \geq 0, i=1, \cdots, l
$$

We solve the minimum of function $\Phi(\omega)=\frac{1}{2}\|\omega\|^{2}=\frac{1}{2}(\omega \cdot \omega)$. So the Lagrange function can be defined as follows:

$$
L(\omega, b, \alpha)=\frac{1}{2}(\omega \cdot \omega)-\sum_{i=1}^{1} \alpha_{i}\left\{y_{i}\left[\left(\omega \cdot x_{i}\right)-b\right]-1\right\}
$$

Where $\omega$ is the weight value of machine learning, $b$ is the threshold of machine learning and $\alpha \geq 0$ is Lagrange coefficient.

At this point the dual problem of the objective function is: the quadratic programming problem of maximization.

$$
W(\alpha)=\sum_{i=1}^{1} \alpha_{i}-\frac{1}{2} \sum_{i, j=1}^{1} \alpha_{i} \alpha_{j} y_{i} y_{j}\left(x_{i} \cdot x_{j}\right)
$$

The condition of constraint is:

$$
\left\{\begin{array}{l}
\alpha_{i} \geq 0, i=1, \cdots, l \\
\sum_{i=1}^{l} \alpha_{i} y_{i}=0
\end{array}\right.
$$

This is an extreme value problem of quadratic function under the inequality constraint, which has a unique solution. According to the Kuhn-Tucker condition, the solution to optimization problem must be met:

$$
\alpha_{i}\left\{y_{i}\left[\left(\omega \cdot x_{i}\right)-b\right]-1\right\}=0, i=1, \cdots, l
$$

Therefore $\alpha_{i}$ which most samples correspond to will be 0 , only a part (usually small part) of the $\alpha_{i}$ is not zero, the corresponding samples are support vectors. While the corresponding classification function also becomes:

$$
f(x)=\operatorname{sgn}\left|\sum_{i} y_{i} \alpha_{i}\left(x_{i} \cdot x\right)-b\right|
$$

The output value of this function is the result of classification.

Be aware of optimal linear classification discriminant function (7) and its solving process (4)、(5) only contain the inner product of this sample vector, if the dot product $\left(x_{i}, x\right)$ is instead of the inner product kernel function $K\left(x_{i}, x\right)$, which is equivalent to transform the original characteristic space $\mathrm{x}$ into a new characteristic space $\Phi(x)$, i.e. $K\left(x_{i}, x\right)=\left(\Phi\left(x_{i}\right), \Phi(x)\right)$.

Through the above analysis, the basic idea of SVM can be summarized as follows: during the classification, for a linear non-separable sample ,first the sample of original space is mapped into a high dimensional characteristic space (also known as the kernel space) through a nonlinear mapping, so the sample becomes linear separable or approximately linear separable in kernel space, then we process the linear classification (or other linear algorithms) in kernel space to achieve the nonlinear algorithm relatived to the original space.

\section{EXPERIMENT OF TARGET RECOGNITION}

\section{A. Experiment process}

According to the feature extraction methods of line spectrum which is analysed above and the basic principles of support vector machine, the specific processing flow of 
underwater target recognition based on the line spectrum feature and SVM in practical application is shown in Fig. 2.

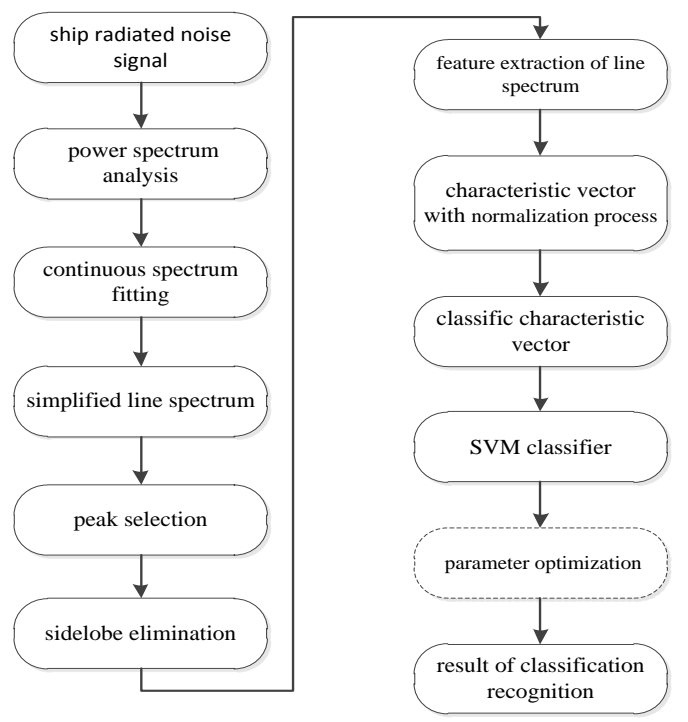

Figure 2. Flow chart of underwater target recognition

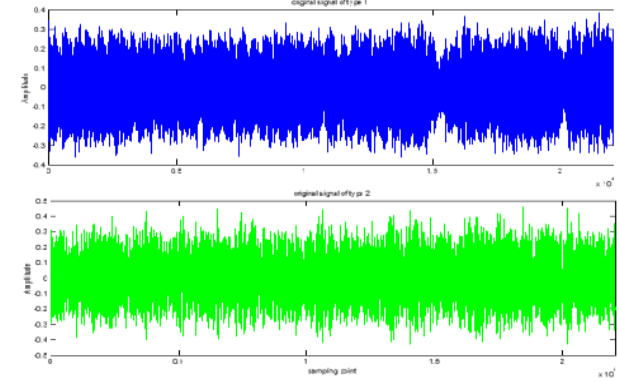

Figure 3. Original signal

\section{B. Characteristic vector extraction}

Here we use two kinds of ship radiated noise signals as an example to process the line spectrum feature extraction, where the frequency of original signal sampling is $22.05 \mathrm{KHz}$, the sampling time is $1 \mathrm{~s}$ and the signal length is 22,050 points. Fig. 3 shows the original waveform of ship radiated noise.

The original signal is processed by the power spectrum estimation, where it uses periodogram, takes 512 points and window function is rectangular window (rectwin). Signal power spectrum estimation and polynomial fitting curve of continuous spectrum are shown in Fig. 4.

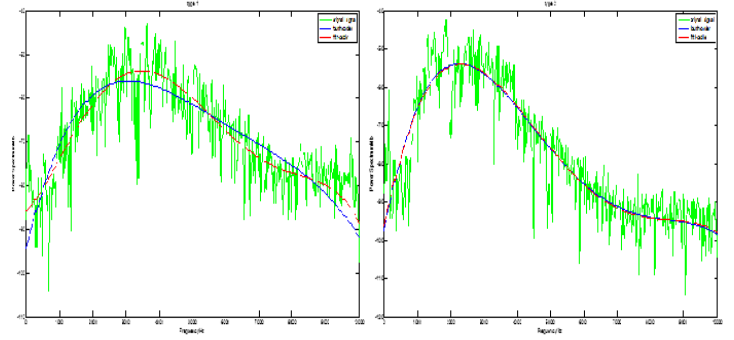

Figure 4. Power spectrum estimation and continuous spectrum fitting of signals

Line spectrum feature of signal is obtained after power spectrum subtracts polynomial fitting continuous spectrum, on this basis, the waveform can be corrected and line spectrum feature is extracted. The following explainations all use fourth-order polynomial fitting as example.

According to the foregoing methods and steps of selecting the peaks and eliminating side lobe, we take $\delta_{\text {gate }}=2 d b 、 \delta_{\text {gate } 2}=3 d b$ and the line spectrum of signal obtained finally is shown in Fig. 5.

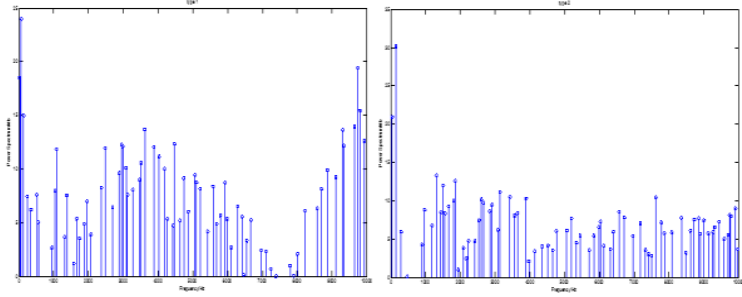

Figure 5. Line spectrum feature of signals

After getting the line spectrum of ship radiated noise, we process feature extraction to constitute the 2K-dimensional original characteristic vector. Specific methods are shown in section 1.4.

The original characteristic vectors of line spectrum which are extracted from different types of ship radiated noises are shown in Table 1. Due to the different ranges of values and the differences in magnitude of each feature extracted, it is necessary to normalize the eigenvalues in order to be ease of classification and prevent the problem that the feature with a larger range of initial value has an excessive weight than this with a smaller range of initial value. The normalization methods in this article are:

For the number of line spectrum: $n_{i}=n_{i} / \sum_{i=1}^{K} n_{i}$

Where $n_{i} 、 n_{i}$ are separately the number of line spectrum of the i-th frequency interval and its normalized value, i.e. the percentage of the converted number of line spectrum.

For the intensity of line spectrum:

$$
S_{i}^{\prime}=n_{i} S_{i} / \sum_{i=1}^{K} n_{i} S_{i}
$$

Where $S_{i} 、 S_{i}$ are separately the average intensity of line spectrum of the i-th frequency interval and its normalized value, i.e. the percentage of the converted intensity of line spectrum.

The schematics of classific characteristic vector with normalization process are shown in Fig. 6, the specific values in Table 2 .

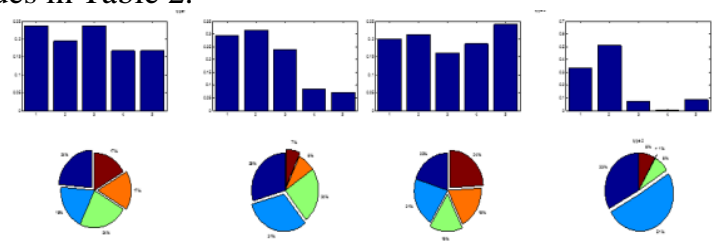

Figure 6. Classific characteristic vector of signals

As can be seen from the above chart: the line spectra distribution of two different kinds of ship radiated noise signals are significantly different, indicating that the physical features of different signals, and these features have relative stability, which can be used to identify the target classification. 


\section{Experiment results}

We adopt the ship radiated noise signals in actual measurement for example. The original sampling is $22 \mathrm{KHz}$, sampling time is $1 \mathrm{~s}$, signal length is 22,050 points.
Two types of signals both have 120 samples, in which, 72 for training and 108 for testing. We classified signals by LIBSVM $^{[11,12]}$ with C-SVM and RBF kernel function, the final results in Table 2 .

TABLE I. ORIGINAL CHARACTERISTIC VECTORS OF LINE SPECTRUM EXTRACTED

\begin{tabular}{|c|c|c|c|c|c|c|c|c|c|c|c|}
\hline \multirow[t]{2}{*}{ Type } & \multirow{2}{*}{$\begin{array}{c}\text { Sequence } \\
1\end{array}$} & \multicolumn{5}{|c|}{ original characteristic vector } & \multicolumn{5}{|c|}{ (fourth-order polynomial fitting, five frequency equant tervals) } \\
\hline & & 9 & 7 & 10 & 5 & 6 & 37.232235 & 14.703231 & 5.464734 & 1.692164 & 2.731653 \\
\hline \multirow[t]{3}{*}{ No.1 } & 2 & 8 & 8 & 8 & 5 & 9 & 26.076860 & 6.653405 & 3.762728 & 2.051720 & 1.665932 \\
\hline & 3 & 7 & 7 & 8 & 6 & 18 & 24.976684 & 8.279081 & 4.595544 & 10.785662 & 5.797021 \\
\hline & 1 & 9 & 8 & 9 & 8 & 10 & 29.088825 & 15.922336 & 1.349101 & 11.293886 & 5.143899 \\
\hline \multirow[t]{2}{*}{ No.2 } & 2 & 8 & 8 & 6 & 7 & 15 & 29.666823 & 0.142869 & 4.652504 & 11.982674 & 7.211109 \\
\hline & 3 & 8 & 6 & 5 & 10 & 15 & 33.497123 & 13.127979 & 1.413591 & 11.166816 & 6.455444 \\
\hline
\end{tabular}

TABLE II. CLASSIFIC CHARACTERISTIC VECTOR WITH NORMALIZATION PROCESS

\begin{tabular}{|c|c|c|c|c|c|c|c|c|c|c|c|}
\hline \multirow[t]{2}{*}{ Type } & \multirow{2}{*}{$\begin{array}{c}\text { Sequence } \\
1\end{array}$} & \multicolumn{10}{|c|}{ normalized characteristic vector (fourth-order polynomial fitting, five frequency equant intervals) } \\
\hline & & 0.243243 & 0.189189 & 0.270270 & 0.135135 & 0.162162 & 0.647504 & 0.198880 & 0.105597 & 0.016349 & 0.031671 \\
\hline \multirow[t]{3}{*}{ No.1 } & 2 & 0.210526 & 0.210526 & 0.210526 & 0.131579 & 0.236842 & 0.657685 & 0.167806 & 0.094900 & 0.032342 & 0.047269 \\
\hline & 3 & 0.152174 & 0.152174 & 0.173913 & 0.130435 & 0.391304 & 0.398611 & 0.132129 & 0.083819 & 0.147542 & 0.237900 \\
\hline & 1 & 0.204545 & 0.181818 & 0.204545 & 0.181818 & 0.227273 & 0.482037 & 0.234536 & 0.022356 & 0.166359 & 0.094712 \\
\hline \multirow[t]{2}{*}{ No.2 } & 2 & 0.181818 & 0.181818 & 0.136364 & 0.159091 & 0.340909 & 0.517703 & 0.002493 & 0.060892 & 0.182966 & 0.235946 \\
\hline & 3 & 0.181818 & 0.136364 & 0.113636 & 0.227273 & 0.340909 & 0.476562 & 0.140078 & 0.012569 & 0.198587 & 0.172203 \\
\hline
\end{tabular}

TABLE III. EXPERIMENT RESULTS OF TARGET RECOGNITION

\begin{tabular}{|c|c|c|c|c|c|c|c|c|c|c|}
\hline $\begin{array}{l}\text { Classification } \\
\text { Method }\end{array}$ & Obj & Rho & $\begin{array}{l}\text { Total } \\
\text { nSV }\end{array}$ & $\begin{array}{c}\text { Training } \\
\text { Time } \\
\text { (ms) }\end{array}$ & $\begin{array}{l}\text { Prediction } \\
\text { Time } \\
\text { (ms) }\end{array}$ & MSE & SCC & $\begin{array}{c}\text { Classification } \\
(\%)\end{array}$ & $\begin{array}{c}\text { Optimized } \\
\text { Parameters } \\
\text { Classification } \\
(\%)\end{array}$ & $\begin{array}{c}\text { KNN-SVM } \\
\text { Classification } \\
(\%)\end{array}$ \\
\hline $\begin{array}{l}3 \text {-order, } \\
5 \text { intervals }\end{array}$ & -104.195563 & 0.173975 & 130 & 53 & 243 & 0.7037 & 0.4663 & 82.4074 & 94.9074 & 84.72 \\
\hline $\begin{array}{l}3 \text {-order, } \\
8 \text { intervals }\end{array}$ & -128.757167 & 0.154975 & 144 & 69 & 276 & 1.4444 & 0.1613 & 63.8889 & 91.6667 & 73.12 \\
\hline $\begin{array}{l}\text { 4-order, } \\
5 \text { intervals }\end{array}$ & -139.584622 & -0.057389 & 144 & 61 & 252 & 1.0370 & 0.2358 & 74.0741 & 79.6296 & 77.78 \\
\hline $\begin{array}{l}\text { 4-order, } \\
8 \text { intervals }\end{array}$ & -140.088805 & 0.017417 & 144 & 72 & 269 & 1.1296 & 0.2213 & 71.7593 & 84.7222 & 72.22 \\
\hline
\end{tabular}

Where, the relationship between classification accuracy and polynomial fitting rank of continuous spectrum or frequency equant interval is shown in Fig. 7. The Fig. shows that: there is a close correlation between classification accuracy and polynomial fitting rank of continuous spectrum or frequency equant interval, the increase in fitting rank and frequency equant interval causes the dimension of input characteristic vector and the amount of computation to increase, which is not necessarily beneficial for classification, we need to find the best combination of both. The optimal parameters in Table 3 are obtained by using LIBSVM software with optimization kits; KNN-SVM uses the algorithm put forward in reference ${ }^{[13,14]}$..

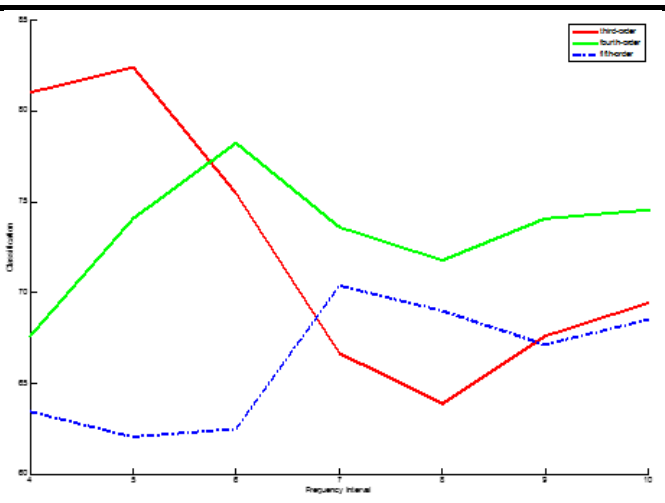

Figure 7. The relationship between classification and fitting rank or frequency interval

The classification experiment results demonstrate that:

- The power spectrum can reflect the basic features of the ship target itself; and the line spectrum feature extracted by power spectrum can truly 
reflect the signal feature and has a certain representative, which can be used as the basis of target identification.

- SVM classification algorithm can effectively classify and the overall recognition rate is higher, the highest is up to $82 \%$ and the lowest is over $63 \%$; The accuracy of classification can be improve more higher if we adopt SVM parameter optimization or KNN algorithm optimization, the highest is up to $94 \%$ and the lowest is close to $80 \%$.

- It is non-significance if we overemphasized the dimension of characteristic vector of ship radiated noise signal, namely the division band of line spectrum, because when it reaches a certain level, the increase in dimension of vector will cause the amount of computation and disturbance to increase, but it is not conducive to the classification, we should make a reasonable match between the two.

\section{CONCLUSIONS}

In the paper, we discuss the theory and method of underwater target recognition of ship radiated noise signal based on signal spectrum analysis and extract and classify the line spectrum characteristic vector from the original noise signal. The simulation experiment results demonstrate that, this method does not need to understand the priori information and feature distribution of the signal, and the dimension of number and intensity characteristic vector of line spectrum extracted is lower and normalization performance is good. The amount of computation is small and speed is fast when it is applied to the classification algorithm, which can effectively identify the type of underwater ship radiated noise. We only aim at basic classification situation of SVM here, namely two types, the next step is to study multi-classes recognition algorithms and how to improve the speed and accuracy of classification recognition.

\section{REFERENCES}

[1] Zhang Xin-hua. A survey on the core techniques in the automatic recognition of sonar targets[J]. ACTA ARMAMENTARII.1998, 19(3): 275-280

[2] DING Yu-wei. Review on passive sonar target recognition[J]. Technical Acoustics. 2004, 23(4): 253-257.

[3] WU Guoqing. Ship radiated-noise recognition(I)--The overall framework, analysis and extraction of line-spectrum[J]. ACTA ACOUTICA. 1998, 23(5): 394-400.

[4] Vapnik, V.N. Statistical learning theory[M]. Beijing: PHEI, 2009.

[5] Nello Cristianini. An introduction to support vector machines[M]. Beijing: China Machine Press, 2004.

[6] ZHANG Xue-gong. Introduction to statistical learning theory and support vector machines[J]. ACTA AUTOMATICA SINICA. 2000, 26(1): 32-42.

[7] ZENG Qing-jun, WANG Fei, HUANG Jian-guo, et al. Research on technique of passive sonar target recognition based on line spectrum feature extraction[J]. Ship Engineering, 2001, (3): 50-54.

[8] ZENG Qing-jun, WANG Fei, HUANG Jian-guo. Technique of passive sonar target recognition based on continuous spectrum feature extraction[J]. JOURNAL OF SHANGHAI JIAOTONG UNIVERSITY. 2002, 36(3): 382-386.

[9] Yao Wu-chuan, YAO Tian-ren. Analyzing classical spectral estimation by MATLAB $[J]$. Journal Huazhong University of Sci \& Tech. 2000, 28(4): 45-47.

[10] YANG Xiang-feng, ZHANG Xiao-ming, SUN Ji-hong. A new power spectrum feature extractive method of ship radiated noise[J]. Torpedo Technology. 2006, 14(1): 35-38.

[11] LIBSVM Software, [2013-10-01]. http://www.cise.ntu.edu.tw/ $\sim$ cjlin/libsvm/ acknowledgements.

[12] Chih-Chung Chang and Chih-Jen Lin. LIBSVM: A library for support vector machines [EB/OL]. [2013-10-01] . http://www.cise. ntu.edu.tw/ cjlin/libsvm.

[13] Xiaoqiao Shen, Yaping Lin. Gene Expression Data Classification Using SVM-KNN Classifier[A]. Proceedings of 2004 International Symposium on Intelligent Multimedia, Video and Speech Processing[C]. Hong Kong, 2004, 149-152.

[14] Liu Jian, XUE Meng. Algorithm and simulation of SVM classifier based on KNN judgment[J]. Ship Electronic Engineering. 2009 29(3): 88-91. 